Fecha de recepción 2019-09-03; fecha de aceptación: 2020-03-01

\title{
Análisis de la exhibición documental de terceros y la disclosure against a person not a party en el proceso civil $^{12}$
}

\section{ANALYSIS OF THE DOCUMENTARY EXHIBITION OF THIRD PARTIES AND THE DISCLOSURE AGAINST A PERSON NOT A PARTY IN THE CIVIL PROCESS}

ELISABET CERRATO GURI

\section{RESUMEN}

Una de las cuestiones que más preocupación genera en el ámbito de la prueba documental es la aportación al proceso de los documentos que no se hallen en disposición de la parte que los quiera utilizar para alcanzar la tutela judicial deseada. Ello sucede cuando esta fuente probatoria se encuentra en manos de otra parte procesal o incluso de un tercero ajeno al proceso judicial. El ordenamiento jurídico español regula esta realidad a través de la exhibición documental en la Ley 1/2000 de Enjuiciamiento Civil (en adelante LEC) ${ }^{4}$, aunque con algunas lagunas y deficiencias. Es por este motivo, y dada la relevancia que supone para el órgano decisor disponer de todos los elementos probatorios para la consecución de la anhelada justicia (art. 1.1 de la Constitución Española, en adelante $\mathrm{CE}$ ), y para las partes utilizar los medios de prueba pertinentes para su defensa (art. 24.2 CE), que hemos querido acudir al derecho comparado para analizar el tratamiento jurídico que recibe esta institución procesal. A tal fin, y por su especial trascendencia en el common law, nos hemos fijado en la institución jurídica de la disclosure, regulada en la Civil Procedure Rules (en adelante CPR) 5 .

En el presente trabajo nos proponemos examinar los elementos esenciales propios de cada una de las referidas instituciones procesales, dedicando especial atención a la aportación documental de terceros en el proceso judicial para, en última instancia, detectar sus puntos de encuentro y desencuentro.

\section{PALABRAS CLAVE}

Prueba, exhibición documental, terceros

\section{ABSTRACT}

One of the most disputed aspects in the documentary evidence scope, is the production, during the legal procedures, of documental evidence when particular pieces of it are not available to the party that wants to use them to achieve judicial protection. This happens when those documents are possesed by a different procedural party or even a non-party. The Spanish legal system regulates this conflict through the documentary exhibition in the Civil Procedure Act, although not without some gaps and shortfalls. This is the reason why we resort to comparative law, in order to analyze the legal treatment that this procedural institution receives. With such goal in sight, and due to its special importance in the common law, we have taken the legal institution of disclosure, regulated in the Civil Procedure Rules, as our subject of comparative analysis.

\footnotetext{
${ }^{1}$ Este trabajo es el resultado de una estancia de investigación realizada en la University of Oxford de mayo a julio de 2019, auspiciada por el profesor Adrian Zuckerman a quien agradecemos las facilidades bibliográficas concedidas.

2 Este trabajo se enmarca en el proyecto I+D "Hacia una nueva regulación de la prueba pericial" (DER2016-7549-P), financiado por el Ministerio de Economía y Competitividad de España, y el Grupo de Investigación Consolidado "Evidence Law" (2017 SGR 1205 ) financiado por la Agència de Gestió d'Ajuts Universitaris i de Recerca de la Generalitat de Catalunya (AGAUR), en ambos como investigador principal el profesor Joan Picó i Junoy.

3 Profesora Agregada de Derecho Procesal, Universitat Rovira i Virgili, Tarragona, España. Correo electrónico: elisabet.cerrato@urv.cat.

${ }^{4}$ Ley N. 1, de 2000. Disponible en https://www.boe.es/buscar/act.php?id=BOE-A-2000-323.

${ }^{5}$ Disponible en http://www.justice.gov.uk/courts/procedure-rules/civil/rules/part31.
} 
The aim of this paper is to examine the essential elements of each of these procedural institutions, with special regard to the non-party documentary contribution in the judicial process, in order to detect points of encounter and disagreement between the above-mentioned regulations.

\section{KEYWORDS}

Evidence, documentary exhibition, person not a party

\section{La exhibición documental en el proceso civil español ${ }^{6}$}

\subsection{El deber de exhibición documental entre partes}

En el transcurso de un proceso civil es posible que una de las partes precise, para respaldar su pretensión, de un documento obrante en poder de otra parte. La LEC regula aquellas situaciones en las que tal documento tenga relación con el objeto del proceso o con la eficacia de los medios de prueba, posibilitando que cualquiera de las partes pueda solicitar de las demás su exhibición. Esta opción legislativa, que está prevista en el art. $328 \mathrm{LEC}^{7}$ como un deber entre partes es, como ha reconocido el Tribunal Supremo ${ }^{8}$, consecuencia directa del principio de buena fe procesal (arts. 11.1 LOPJ y 247 LEC) ${ }^{9}$ e impone a las partes la obligación de colaboración para la correcta resolución de la controversia (fundamentada en el art. $118 \mathrm{CE}$ ). Sin embargo, este propósito del legislador no ha alcanzado su real materialización pues deja de concretar los sujetos contra los que puede dirigirse la exhibición documental; tampoco especifica los extremos que debe contener la inicial solicitud para que se lleve a cabo la referida exhibición ni la forma que debe adoptar la resolución por la que se acuerde la exhibición documental; finalmente, olvida asimismo el legislador determinar las consecuencias ante la negativa de la parte requerida a aportar los documentos requeridos.

La deficiente regulación de este precepto normativo hace ineludible que, para una mejor interpretación de la norma, debamos acudir al estudio de la doctrina judicial y científica. Ello nos permitirá ver cómo se han solucionado, en la práctica, los principales problemas que sobre esta materia se han suscitado.

Como una primera cuestión, nos centraremos en los sujetos contra quienes puede solicitarse la exhibición documental. Al respecto, estamos de acuerdo con Abel Lluch al considerar que más allá de la contraparte la solicitud también puede dirigirse a los colitigantes con intereses contrapuestos ${ }^{10}$. A mayor abundamiento, y aunque nada diga la literalidad del art. 328 LEC, como no podía ser de otra manera, de su redacción se desprende que esta petición deberá presentarse ante el órgano judicial, y así lo ha confirmado la doctrina científica y judicial ${ }^{11}$.

También es preciso aclarar qué extremos de la solicitud deben ser objeto de consideración por parte el tribunal para su admisión. De la interpretación conjunta que la jurisprudencia ha hecho de los apartados 1 y 2 del art. 328 LEC deriva que para que sea admitida, el tribunal deberá valorar la concurrencia de los siguientes requisitos: "(i) justificar que el documento no se halla a su disposición y la imposibilidad de obtenerlo salvo que medie cooperación de la requerida; (ii) acreditar y justificar que el documento se refiere al objeto del proceso o a la eficacia de los

\footnotetext{
${ }^{6}$ La problemática práctica que plantea esta cuestión ha sido rigurosamente examinada por CASANOVA (2019), pp. 766-773.

${ }^{7}$ No obstante, para los procesos seguidos por infracción de un derecho de propiedad industrial o de un derecho de propiedad intelectual, cometida a escala comercial, deberá estarse a la concreta regulación del apartado tercero del art. 328 LEC, según el cual: "la solicitud de exhibición podrá extenderse, en particular, a los documentos bancarios, financieros, comerciales o aduaneros producidos en un determinado período de tiempo y que se presuman en poder del demandado. La solicitud deberá acompañarse de un principio de prueba que podrá consistir en la presentación de una muestra de los ejemplares, mercancías o productos en los que se hubiere materializado la infracción. A instancia de cualquier interesado, el tribunal podrá atribuir carácter reservado a las actuaciones, para garantizar la protección de los datos e información que tuvieran carácter confidencial”. Ley № 1, de 2000 .

${ }^{8}$ STS 644/2017, Sala de lo Civil, de 24 de noviembre, f.j. 10 (ECLI: ES:TS:2017:4119).

${ }^{9}$ Sobre la buena fe procesal véase PICÓ (2012).

${ }^{10}$ ABEL (2010), p. 115

11 Véase por todos PICÓ (2014), p. 3948. Por su parte véase también la SAP Barcelona, 848/2017, de 18 de diciembre, f.j. 2 o (ECLI:ES:APB:2017:11973).
} 
medios de prueba; y (iii) aportar copia del documento o, en su defecto, indicar en los términos más exactos posibles su contenido"12.

Otra cuestión que no esclarece expresamente el precepto analizado es la forma de la resolución que acuerde dicha exhibición documental. En nuestra opinión, la trascendencia de esta ausencia es menor por cuanto entendemos que al tratarse de admisión probatoria será un auto, tal y como se prevé en la genérica regulación del art. 206.1.2 ${ }^{\mathrm{a}}$ del mismo texto legal ${ }^{13}$. Este auto debería indicar al requerido un plazo razonable para llevar a cabo la exhibición, así como advertirle de las consecuencias de su negativa injustificada (art. 329 LEC) ${ }^{14}$.

Una vez acordada la exhibición documental, se requerirá a la parte afectada por la resolución para que aporte voluntariamente los documentos relacionados en el auto ${ }^{15}$. Ahora bien, ¿está la parte obligada a atender tal reclamación? La respuesta debe ser negativa pues tal y como ha reconocido parte de la doctrina científica en realidad estamos ante una carga y no un deber de la parte, que podrá optar por no hacerlo, incluso de manera injustificada ${ }^{16}$. En este último caso, deberá estarse, de entrada, a las consecuencias del art. 329 LEC, que otorga al órgano judicial una doble posibilidad: en primer lugar, atribuir valor probatorio a la copia simple presentada por el solicitante de la exhibición o a la versión que hubiera dado del contenido del documento, si bien debiendo tener en consideración las restantes pruebas ${ }^{17}$; o, en segundo lugar, y cuando entienda que las características del concreto proceso así lo precisen, formular un segundo requerimiento, mediante providencia, para que quien no lo hizo injustificadamente, aporte tales documentos ${ }^{18}$. Pero ¿qué sucede si se sigue incumpliendo este segundo requerimiento judicial? Aunque el art. 329.2 LEC no lo haya especificado, debe entenderse que serán de aplicación las mismas consecuencias que las previstas en su apartado anterior ${ }^{19}$.

A pesar de todo lo expuesto, en última instancia debe clarificarse que esta actitud de la parte no comporta la inmediata aplicación del art. 329 LEC pues esta es facultativa y no preceptiva para el órgano judicial ${ }^{20}$. Siendo esto así, a nuestro juicio, esta opción del legislador da cobertura al requerido para arriesgarse a no presentar el documento injustificadamente porque existe la posibilidad de que el tribunal acabe optando por no dar valor probatorio a la copia o al contenido del documento dado por la contraparte; o bien apostar por esgrimir motivos (económicos, por ejemplo) que justifiquen de manera suficiente la reticencia a exhibir los documentos.

\subsection{La exhibición de documentos por terceros}

\subsubsection{Regla general}

Asentadas las anteriores premisas, nos planteamos si es posible que la exhibición documental pueda extenderse a terceros que, a pesar de no estar vinculados al proceso judicial, tengan en su poder documentos que pueden ser determinantes para el esclarecimiento de los

\footnotetext{
12 STS 644/2017, Sala de lo Civil, de 24 de noviembre, f.j. 10 (ECLI: ES:TS:2017:4119). En términos parecidos se pronuncia la SAP Barcelona, 848/2017, de 18 de diciembre, f.j. 2ㅇ (ECLI:ES:APB:2017:11973).

${ }^{13}$ Confróntese PICÓ (2014), p. 3948.

${ }^{14}$ Confróntese ABEL (2010), p. 117, con cita a la "SAP Murcia, secc. 5a , de 22 de enero de 2007, fto.jco. 3 (JUR 2007\266012)".

${ }^{15} \mathrm{El}$ art. $331 \mathrm{LEC}$ contempla la posibilidad de que la parte que debe realizar la exhibición no quiera desprenderse del documento para su incorporación a los autos, en cuyo caso, podrá solicitar que el Letrado de la Administración de Justicia extienda testimonio del documento en sede judicial. Ley № 1, de 2000.

${ }^{16}$ Así lo justifica PICÓ (2014), p. 3948, según el cual "más que un deber el art. 328 LEC recoge una carga pues, como indicaremos, tan solo prevé como consecuencia de su infracción la posibilidad de que la parte 'cargada' vea desestimada su petición al dar se valor a la copia -o versión- del documento cuya aportación se solicita".

17 STS 644/2017, Sala de lo Civil, de 24 de noviembre, f.j. 10 (ECLI: ES:TS:2017:4119).

18 A estas consecuencias se ha referido la reciente SAP de Barcelona (Sección 15a)), 423/2018, de 25 de junio, f.j. 60 (ECLI:ES:APB:2018:6540)

${ }^{19}$ De esta misma opinión, PICÓ (2014), p. 3949.

${ }^{20}$ SAP de Bilbao (Sección 4a), 366/2017, de 17 de mayo, f.j. 4o (ECLI: ES:APBI:2017:997); SAP de Salamanca (Sección 1a), 36/2012, de 30 de enero, f.j. 3o (ECLI: ECLI:ES:APSA:2012:37); y STS 479/2008, Sala de lo Civil, de 3 de junio, f.j. 60 (ECLI: ES:TS:2008:4309).
} 
hechos y, en consecuencia, su aportación al proceso interese a alguna de las partes. Sin duda, la respuesta debe ser afirmativa, en virtud del art. 330 LEC $^{21}{ }^{22}$, a pesar de su deficiente e incompleta regulación ${ }^{23}$. Pero ¿quién merece la consideración de tercero? Sobre esta cuestión, la LEC, en su art. 330.2, ha clarificado que no tendrán tal consideración los titulares de la relación jurídica controvertida o de las que sean causa de ella, aunque no figuren como partes en el juicio ${ }^{24}$. En consecuencia, y por exclusión, tendrán la condición de tercero a estos efectos quienes no reúnan las anteriores características ni sean parte procesal.

En particular, para que proceda la exhibición documental por un tercero deberán cumplirse los siguientes requisitos:

a) que la solicitud sea a instancia de la parte que pretenda la exhibición documental, lo que excluye que pueda acordarse de oficio ${ }^{25}$;

b) que el documento esté en propiedad del tercero, de manera que éste "no está obligado a exhibir o aportar lo que no tiene ${ }^{\prime 26}$. Además, es preciso en este contexto matizar que el término "propiedad" hace referencia a la "disponibilidad" del documento y no a su titularidad ${ }^{27}$, por lo que en todo caso dicha exhibición deberá requerirse al tercero que tenga el documento en su poder; $y$,

c) que el conocimiento del documento resulte trascendente a los fines de dictar sentencia, lo que tendrá que valorar el tribunal en cada concreto $\operatorname{caso}^{28}$, más allá de la pertinencia o utilidad de la prueba ${ }^{29}$.

Solo cuando concurran los anteriores requisitos, el órgano judicial dictará una providencia ordenando la comparecencia personal del tercero con la finalidad de oírle para decidir sobre la exhibición ${ }^{30}$. En la comparecencia, el tribunal acordará o denegará la exhibición solicitada, siendo en todo caso irrecurrible esta decisión, al margen de que quien tuviere interés en ello, pueda reproducir su petición en segunda instancia (art. 330.1.2 LEC).

Ante el requerimiento de exhibición de documentos, el tercero puede adoptar dos actitudes distintas: exhibirlos o no. Sin embargo, el legislador únicamente se ha centrado en el primero de los supuestos (art. 330.1.3 LEC), dejando de regular las consecuencias de su no exhibición, a diferencia de lo que sucede con la exhibición documental entre partes. Así, en el caso de exhibición voluntaria, el tercero podrá, o bien presentarlos en sede judicial, o bien exigir que tal exhibición tenga lugar en su domicilio, en cuyo caso el Letrado de la Administración de Justicia lo testimoniará ${ }^{31}$.

Ahora bien, ¿̇y si no los exhibe? De la propia redacción del precepto citado, parece que el legislador no ha querido otorgar carácter obligatorio a la exhibición de documentos por terceros al no contemplar expresamente consecuencia alguna a la negativa a su exhibición. Y, de hecho, así lo ha confirmado la doctrina judicial mayoritaria, debiéndose traer a colación, por su claridad expositiva, la SAP de Huelva (Sección 1á) de 26 de octubre de $2010^{32}$, en atención a la cual "[...]

\footnotetext{
${ }^{21}$ SAP Barcelona (Sección 4a), 848/2017, de 18 de diciembre, f.j. 2으 (ECLI:ES:APB:2017:11973).

22 En el ordenamiento jurídico chileno, la exhibición de documentos por terceros se encuentra regulada en el art. 349 (338) del Código de Procedimiento Civil, Ley No 1552, de 1902. Disponible en https://www.leychile.cl/Navegar?idNorma=22740\#documentos0.

${ }^{23}$ En esta línea, véase también ABEL (2010), pp. 124-129.

${ }^{24}$ Esto sucede en la SAP de Oviedo (Sección 6a) ), 126/2014, de 2 de junio, antecedente de hecho 20 (ECLI:ES:APO:2014:1419).

${ }^{25}$ A pesar de ello, PICÓ (2014), p. 3949, señala que "cabe la posibilidad de que se decrete esta actividad probatoria ex oficio iudicis" por la vía de las diligencias finales del art. 435.2 LEC.

${ }^{26}$ SAP de Barcelona (Sección 11a) ), 211/2015, de 23 de julio, f.j. 1o (ECLI:ES:APB:2015:8022).

${ }^{27}$ Al respecto especialmente crítico se muestra ABEL (2010), p. 126.

${ }^{28}$ Sobre esta necesidad se pronuncia la SAP de Granada (Sección 5ạ), 412/2012, de 26 de octubre, f.j. 10 (ECLI:ES:APGR:2012:2300).

29 De esta opinión son PICÓ (2014), p. 3949 y ABEL (2010), p. 125.

${ }^{30}$ En caso de no comparecer, parte de la doctrina científica ha considerado que esta actitud del tercero podría llegar a tener como consecuencia la imposición de una condena penal en aplicación del art. $556 \mathrm{CP}$, relativo al delito de desobediencia a la autoridad. A esta consecuencia se han referido PICÓ (2014), p. 3950 y ABEL (2010), p. 127.

${ }^{31}$ En este caso, sería asimismo de aplicación el art. 331 LEC, Ley № 1, de 2000, de manera que cuando el tercero no quisiera desprenderse del documento para su incorporación a los autos podría -del mismo modo que se dispone la norma para las partessolicitar que el Letrado de la Administración de Justicia extendiera testimonio del documento en sede judicial.

32 SAP de Huelva (Sección 1a), 194/2010, de 26 de octubre, f.j. 10 (ECLI:ES:APH:2010:682). En esta misma línea, véase también la SAP de A Coruña (Sección 6ạ), 157/2005, de 22 de marzo, f.j. 10 (JUR 2006/93886). En sentido contrario se ha pronunciado la SAP de
} 
la prueba documental propuesta de nuevo fue admitida en primera instancia y va referida a la obtención de documentos de un tercero (...) que respondió con los datos que dice tener en su poder. Los arts. 330 y 331 LEC no permiten en estos casos requerirlo de nuevo bajo apercibimiento de desobediencia a la autoridad judicial, como postula la parte recurrente. Esto solo sería posible en caso de entidades públicas, cuya obligación de aportación al proceso expresamente prevé el art. 332 LEC'33.

Finalmente, es preciso indicar que la aportación documental no se limitará a la estricta incorporación a los autos del documento requerido (o, en su caso, de su testimonio, de acuerdo con el art. 331 LEC) sino que deberá además trasladarse a las partes, inclusive a aquellas que no hubieran solicitado su exhibición y que presumiblemente desconocieran su contenido. De no ser así, "patente resultaría la indefensión que se produciría a las partes, el potencial desequilibrio y la infracción del 'principio de igualdad de armas procesales', en perjuicio de la parte no solicitante, en aquellos supuestos en que el contenido del documento si sea conocido por la adversa"34.

\subsubsection{Especial mención al deber de las entidades oficiales}

A pesar de lo indicado en el apartado anterior, el legislador ha previsto expresamente una particularidad al régimen general de la exhibición documental de terceros, otorgando un tratamiento especial a los terceros que tengan la consideración de entidades oficiales, a saber: dependencias del Estado, Comunidades Autónomas, provincias, Entidades locales y demás entidades de Derecho público (art. 332.1 LEC); así como también entidades y empresas que realicen servicios públicos o estén encargadas de actividades del Estado, de las Comunidades Autónomas, de las provincias, de los municipios y demás Entidades locales (art. 332.2 LEC).

A diferencia de lo que sucede con carácter general para el resto de los terceros, en esta ocasión las entidades oficiales sí que tienen la obligación ${ }^{35}$-esto es, no podrán negarse- de atender a la exhibición judicialmente requerida. Al respecto, debe aclararse que la norma procesal no se ha limitado únicamente a la exhibición de los documentos que obren en sus dependencias y $\operatorname{archivos}^{36}$, sino que ha ido más allá, exigiéndoles también la expedición de las certificaciones y testimonios solicitados por los tribunales.

Sin embargo, como no podría ser de otra manera, puede excepcionarse de este deber la exhibición de la documentación legalmente declarada o clasificada como de carácter reservado o secreto. Para que el tribunal así lo acuerde, corresponderá a la entidad oficial la justificación de tal consideración ${ }^{37}$.

\footnotetext{
Granada (Sección 5a), 412/2012, de 26 de octubre, f.j. 1으 (ECLI:ES:APGR:2012:2300), al establecer que "[...] Solo debe añadirse que la prueba documental interesada por la entidad actora en la audiencia previa, y admitida, se hizo correctamente al amparo del art. 330 LEC, por cuanto que Xtra Telecom tenía la condición de tercero no litigante que estaba obligado a la exhibición documental cuando, pedida por la parte demandante, el juzgador consideró que era transcendente para el dictado de la sentencia, así como ocurrió".

33 Para cierta doctrina autorizada, la inconcreción de la norma en este punto podría asimismo llevar a considerar la incurrencia del tercero en un delito de desobediencia a la autoridad (art. 556 CP, Ley Orgánica № 10, de 1995). En esta línea se manifiesta PICÓ (2014), p. 3950, quien además ha puntualizado que "mucho más difícil de justificar sería ordenar la entrada y registro en el domicilio del tercero para que pueda adquirirse el documento en cuestión, pues esta medida supondría restringir el derecho fundamental a la inviolabilidad domiciliaria. No obstante, en supuestos muy excepcionales, podrá decretarse esta medida, ya que no podemos olvidar la existencia de otros derechos en colisión al anteriormente indicado, y muy singularmente, el derecho a la prueba del art. 24 CE".

${ }^{34}$ SAP de Palencia (Sección 1a), 247/2010, de 20 de septiembre, f.j. 2o (ECLI:ES:APP:2010:450).

${ }^{35}$ Lo reconoce expresamente la SAP de Huelva (Sección 1a) ), 194/2010, de 26 de octubre, f.j. 1ํ (ECLI:ES:APH:2010:682).

${ }^{36}$ Por lo tanto, no podrá exigirse a una entidad oficial que certifique sobre extremos o documentos que no ha elab orado o no tiene en sus archivos, tal y como señala la SAP de Badajoz (Sección 3a), 88/2003, de 18 de febrero, f.j. 20 (JUR 2003\162412).

${ }^{37}$ Ello sucede en la SJMercantil núm. 7 de Barcelona, 357/2011, de 16 de diciembre, f.j. 9o (ECLI:ES:JMB:2011:205).
} 


\section{El deber de disclosure}

\subsection{Aproximación a la institución procesal ${ }^{38}$}

En su caso, el ordenamiento jurídico inglés prevé el deber de revelación de ciertos documentos (disclosure) por las partes durante el transcurso del proceso civil para que posteriormente sean objeto de inspección por las otras partes ${ }^{39}$. Esta exigencia es una de las primeras obligaciones que las partes deben cumplir en el marco de un proceso judicial ${ }^{40}$, que adquiere especial protagonismo en el ámbito de la prueba documental ${ }^{41}$.

En virtud de la rule 31.2 CPR - "a party discloses a document by stating that the document exists or has existed" - la disclosure puede definirse como el simple proceso que debe seguir una parte para informar a la otra de que un documento existe o ha existido. Se pretende, con ello, el intercambio de pruebas documentales necesarias para el litigio, lo que se consigue a través del envío, por una de las partes, de una lista de los documentos que obran en su poder a las otras partes (disclosure o revelación) ${ }^{42}$. Quien reciba la lista, deberá indicar, de los documentos relacionados por la otra parte, cuáles desea ver (inspection o inspección) ${ }^{43}$.

El fundamento de esta institución procesal radica en la necesidad de que las partes se intercambien la información sobre la que pretenden sostener sus pretensiones, incluso cuando pudiera perjudicarles ${ }^{44}$. En este contexto, la doctrina británica ha querido precisar que el deber de aportar los documentos se basa en la premisa de que en el proceso deben ponerse "the cards on the table", para que sea más justo. Adicionalmente, señala que los documentos que deben aportarse son aquellos que tengan realmente valor para el proceso, sin que su coste de aportación afecte a la equidad, eficiencia y proporcionalidad ${ }^{45}$.

\section{2. ¿Qué debe entenderse por documento a efectos de disclosure?}

La respuesta a esta pregunta nos la da el apartado 4 de la rule 31 CPR, que debe complementarse con la regla 33.6 del mismo código procesal. Según esta regla, es documento "cualquier cosa en la que se registra información de cualquier clase" ('document' means anything in which information of any description is recorded), por lo que estamos ante un concepto amplio de documento que no se restringe estrictamente al formato papel ni tampoco a los originales, extendiéndose también a las copias y a cualquier otro soporte donde pueda

\footnotetext{
${ }^{38}$ Siguiendo DE PRADA y MUÑOZ (2014), p. 110, se denominaba discovery al "procedimiento previo al juicio por el cual una parte de un procedimiento civil obtenía forzosamente documentos o información de la otra parte que resultaban de especial interés para el litigio". Sin embargo, una de las consecuencias de la entrada en vigor de las CPR fue la modificación de esta institución procesal, que pasa a denominarse disclosure, con el propósito de reducir la obligación de las partes a "afirmar la existencia de un documento" surgiendo, solo tras su comprobación, "un derecho a inspeccionar dicho documento". A mayor abundamiento, HOLLANDER (2015), p. 115, concreta que Lord Wolff en el "Access to Justice", esto es, el informe que dio lugar a las CPR, destacó que uno de los problemas de la litigación era el excesivo gasto de tiempo y dinero en el discovery. Ello explica que una de las principales reformas de las CPR haya afectado al discovery, cambiando su nombre a disclosure, con el objetivo de reducir la cantidad de documentos objeto de disclosure, evitando de este modo la revelación de un volumen importante de documentos innecesarios.

Por su parte, la influencia del discovery al ordenamiento procesal chileno ha sido objeto de estudio por parte de la doctrina procesal chilena, destacando PEÑA (2017).

${ }^{39}$ De conformidad con la regla 31.11 CPR, este deber se mantiene durante todo el proceso hasta su conclusión. Ello implica que en caso de que una parte tenga noticia de cualquier documento relevante para el juicio, deberá inmediatamente notificarlo al resto de partes. A pesar de ello, existen excepciones al deber de disclosure de las partes (por ejemplo, cuando el documento puede perjudicar el interés público - regla 31.19); así mismo, el tribunal podrá limitar o dispensar este deber cuando lo considere oportuno (regla 31.5.1.b); e incluso, las propias partes podrán pactar por escrito esta dispensa o límite (regla 31.5.1.c).

${ }^{40}$ CUNNINGHAM-HILL y ELDER (2013), p. 292.

${ }^{41}$ CUNNINGHAM-HILL y ELDER (2013), p. 111.

42 Respecto del contenido de la lista, véase BROWNE y CATLOW (2014), pp. 177-178.

${ }^{43}$ Sobre esta cuestión, véase BLACKIE y BATES (2014), p. 219. A mayor concreción, ZUCKERMAN (2013), p. 723; y SMITH (2015), p. 870 , coinciden al destacar que la disclosure tiene lugar en dos fases, debiéndose señalar que, en la primera, las partes intercambian el listado de documentos, siendo en la segunda donde se permite la inspección de los anteriores documentos revelados.

${ }^{44}$ Véase DE PRADA y MUÑOZ (2014), p. 110.

${ }^{45}$ Véase CUNNINGHAM-HILL y ELDER (2013), p. 292.
} 
haber información de cualquier tipo, siendo de destacar los documentos electrónicos ${ }^{46}$-no solo el correo electrónico sino también otras comunicaciones electrónicas, documentos de texto y bases de datos-; los documentos fácilmente accesibles desde los sistemas informáticos y otros dispositivos electrónicos y medios de comunicación; aquellos que se almacenan en servidores y back-up systems, inclusive los que han sido borrados ${ }^{47}$.

\section{Disclosure against a person not a party}

\subsection{Contextualización}

Partiendo del importante papel que juega el deber de disclosure de las partes, y al hilo del propósito del presente estudio, ¿puede este deber exigirse también a terceros ajenos al proceso judicial? Esta cuestión debe responderse afirmativamente tal y como ocurre en el caso español.

En el proceso civil inglés también puede suceder que las partes tengan la necesidad de obtener documentos que estén en disposición de terceros para utilizarlos como prueba en beneficio del proceso. Siguiendo a Zuckerman ${ }^{48}$, tradicionalmente esta posibilidad no se contemplaba en las normas de la discovery. Si bien, con la entrada en vigor de las CPR se regulan dos procedimientos distintos para obtener documentos de terceros: el witness summons to produce documents (rule $34 \mathrm{CPR})^{49}$ y la order for disclosure against non-parties (rule 31.17 $(\mathrm{CPR})^{50}$, es al segundo de ellos al que dedicamos nuestra atención, por cuanto se extiende más allá de la estricta reproducción de documentos por un tercero en el acto del juicio utilizándose, además - como ocurre con la standard disclosure- para exigir a dicho tercero la búsqueda de documentos con la finalidad de aportarlos al proceso.

En concreto, la regla 31.17 CPR, que impone el deber de disclosure a terceros ajenos al conflicto que ha dado lugar al proceso judicial se aplica, a diferencia de lo que sucede con el deber de disclosure entre partes, exclusivamente una vez iniciado dicho proceso ${ }^{51}$ y se extiende a toda clase de acciones y procesos ${ }^{52}$. Esta diferencia es clave y hay que destacarla, pues gran parte del disclosure entre partes se produce antes de iniciar el procedimiento judicial como parte de los pre-action protocols $s^{53}$ y tanto es así que el juez exigirá que se le demuestre que estos efectivamente se siguieron ${ }^{54}$.

\footnotetext{
${ }^{46}$ Debemos subrayar que la disclosure de documentos electrónicos se denomina e-disclosure (electronic disclosure) y se encuentra regulada en la PD 31B (Disclosure of Electronic Documents). A mayor abundamiento véase ZUCKERMAN (2013), pp. 739-743.

${ }^{47}$ A ello se han referido ZUCKERMAN (2013), p. 723; CUNNINGHAM-HILL y ELDER (2013), p. 292; BROWNE y CATLOW (2014), p. 166; y SMITH (2015), p. 835.

48 ZUCKERMAN (2013), p. 764.

${ }^{49} \mathrm{El}$ witness summons va dirigido a personas (terceros) que tengan en posesión documentos relevantes para que asistan al acto del juicio y declaren sobre dichos documentos. Para un análisis más pormenorizado de esta institución véase ZUCKERMAN (2013), pp. 765-767; y MATTHEWS y MALEK (2017), pp. 293-319.

${ }^{50}$ La distinción entre la disclosure order against a non-party y el witness summons encuentra reflejo en el caso Tajik Aluminium Plant vs Hydro Aluminium AS [2005] EWCA Civ 1218.

${ }^{51}$ Así se han encargado de indicarlo GREENE (1999), p. 249; y, HILL et al. (2005), p. 238.

52 Inicialmente, la posibilidad de solicitar una orden de disclosure contra un tercero se extendía únicamente en aquellos procesos de lesiones personales y casos de muerte. De ello se han hecho eco, entre otros, DI MAMBRO (2019), p. 884; ASHFIELD et al. (2015), p. 846; y GREENE (1999), p. 249.

${ }^{53}$ La disclosure o revelación temprana de documentos relevantes entre las partes es una característica importante del proceso civil inglés. En cualquier disputa se espera que las partes cumplan con un protocolo específico previo a la acción, o en caso de no existir, actúen de conformidad con lo establecido en la Practice Direction Pre-Action Conduct and Protocols de las CPR, que determina la conducta a seguir por las partes previo inicio del proceso judicial en relación al intercambio de información suficiente esencialmente para comprender la posición de la otra parte e incluso, evitar el inicio del proceso judicial o, en su caso, decidir cómo avanzar pudiendo optar por la resolución de conflicto a través un ADR (Alternative Dispute Resolution) (https://www.justice.gov.uk/courts/procedure-rules/civil/rules/pd_pre-action_conduct).

${ }^{54}$ Entre la doctrina británica que se ha hecho eco de esta consecuencia destacamos DI MAMBRO (2019), p. 803. Sobre los pre-action protocols, a mayor abundamiento, véase HOLLANDER (2015), pp. 1-5; y CUNNINGHAM-HILL y ELDER (2013), p. 318.
} 


\subsection{Finalidad del deber de disclosure de terceros}

Como hemos tenido la oportunidad de apuntar, el deber de disclosure previsto en el ordenamiento jurídico inglés obliga a las partes del proceso judicial a revelar, para su posterior inspección, todos los documentos que estén o hayan estado en su poder y que puedan resultar de utilidad para el proceso. El criterio que se sigue es que este deber recaiga en las partes procesales, si bien, y siempre y cuando se cumplan los requisitos legalmente contemplados en la rule 31.17 CPR, podrá extenderse también a un tercero ajeno a la contienda, de modo que sea éste quien tenga la obligación de revelación. En consecuencia, el deber de disclosure de un tercero es una excepción a la anterior regla general ${ }^{55}$. A pesar de ello, la doctrina científica ha querido subrayar la similitud entre el deber de disclosure de las partes y el de los terceros, con la salvedad que la carga impuesta al tercero es más limitada que la atribuida a las partes ${ }^{56}$. Sin embargo, y debido a la observancia de los pre-action protocols, el juicio propiamente dicho se centrará más bien en este tipo de exhibición que en la de partes, salvo que se haya alegado algún género de privilegio que escude la reticencia a exhibir.

En particular, el procedimiento de non-party disclosure tiene por finalidad que las partes del proceso que ya se ha iniciado tengan alcance a la revelación de documentos de terceros ajenos al mismo cuando tales documentos puedan ser de utilidad para la resolución del conflicto. El supuesto más habitual de aplicación de esta figura es cuando, en cumplimiento de su deber de disclosure, una de las partes identifique entre los documentos relacionados con la causa, aquellos que ya no se encuentren en su poder por estar en posesión de un tercero. Esta circunstancia abre la posibilidad a que las otras partes puedan requerir al tercero la entrega voluntaria de una copia del documento y solo si el tercero rechaza tal petición, podrán las partes instar una orden judicial de disclosure contra el tercero ${ }^{57}$.

No obstante, el tribunal también podrá ordenar el deber de non-party disclosure a un testigo (witness) con la finalidad de que presente aquellos documentos relevantes que obren en su poder con anterioridad al acto del juicio, evitando de este modo tener que esperar hasta ese momento procesal para conocer la existencia y el contenido de dichos documentos. En este caso, se pone de relieve la utilidad de esta institución procesal, ya que la temprana revelación puede evitar la necesidad de suspender el juicio ${ }^{58}$.

\subsection{Procedimiento para la adopción de la orden judicial}

\subsubsection{La solicitud}

Quien pretenda pedir al órgano judicial que requiera a un tercero para que cumpla con su deber de disclosure (applicant), presentará una solicitud contra la persona que deba realizar la revelación documental (respondent) ${ }^{59}$. En la solicitud el applicant no solo deberá identificar los documentos objeto de disclosure sino también justificará los motivos por los cuales tales documentos son necesarios para resolver el litigio, todo ello en cumplimiento del apartado tercero de la rule $31.17 \mathrm{CPR}^{60}$. Además, dicha solicitud deberá acompañarse de prueba suficiente de acuerdo con lo exigido en el apartado segundo de la rule 31.17 CPR.

\footnotetext{
${ }^{55}$ O'HARE y BROWNE (2013), p. 520.

${ }^{56}$ Así lo afirma ZUCKERMAN (2013), p. 765.

${ }^{57}$ Confróntese BROWNE y CATLOW (2014), p. 180.

${ }^{58}$ Esta cuestión ha sido objeto de reflexión por DI MAMBRO (2019), p. 918.

${ }^{59}$ Sobre a quién corresponde la posición de respondent véase GREENE (1999), p. 249.

${ }^{60}$ GREENE (1999), p. 249.
} 


\subsubsection{Requisitos para su emisión}

Para que el tribunal acuerde la orden de disclosure deberán inicialmente cumplirse los dos requisitos del apartado tercero de la rule $31.17 \mathrm{CPR}^{61}$, a saber:

a) que sea probable (likely) que los documentos cuya revelación se solicita respalden la posición del solicitante (applicant) o afecten negativamente a cualquiera de las otras partes del proceso; y

b) que la revelación de documentos sea necesaria para alcanzar un resultado justo (to dispose fairly of the claim) o para ahorrar costes.

Especial mención merece la primera de las condiciones legalmente señaladas, pues el órgano judicial deberá valorar la probabilidad -likely- de que el documento afectado por la disclosure sirva para apoyar o perjudicar la posición de alguna de las partes inmersas en el proceso. De modo que, de no existir esta probabilidad la orden correrá el riesgo de ser rechazada. En este contexto la doctrina judicial ha matizado que el término "likely" se corresponde con la expresión "may well" o quizás, y no debe confundirse con "more probable than not", teniendo en cuenta que esta última expresión hace referencia a la posibilidad, aunque sea remota, de que el documento sea útil para el proceso judicial ${ }^{62}$. Todavía en este ámbito, para determinar la utilidad real del documento afectado por la disclosure, el tribunal deberá examinar cada documento en su contexto y en relación con el resto de los documentos objeto de revelación, pues el análisis individual y aislado de tales documentos puede conducir a una errónea conclusión del juez respecto de su utilidad en el proceso ${ }^{63}$.

Con todo, el cumplimento de las anteriores condiciones legales no comportará la automática adopción de la orden de disclosure, sino que será imprescindible que el tribunal tenga además en consideración la proporcionalidad de lo que se pide en relación con el supuesto concreto - pues de no respetarse, ello puede ser un motivo de oposición a la revelación del documento ${ }^{64}-$. Solo tras haber valorado los anteriores requisitos y circunstancias propias del caso, el tribunal estará en condición de decidir, en virtud de la discrecionalidad que tiene atribuida para ello, si otorga la orden de revelación solicitada ${ }^{65}$.

\subsubsection{Contenido de la orden judicial}

La orden que acuerde el deber de disclosure a un tercero debe contener necesariamente los siguientes dos extremos, regulados en la rule 31.17 (4) CPR ${ }^{66}$. En primer lugar, indicará al tercero que concrete los documentos o clases de documentos objeto de revelación; $y$, en segundo lugar, le requerirá para que especifique en el momento de la revelación, en su caso, si los documentos ya no están en su poder o, aun teniéndolos, considera que sobre ellos tiene un derecho o un deber de no permitir su inspección. Asimismo, el órgano judicial tendrá la facultad de incluir en su resolución la hora y el lugar en que debe llevarse a cabo la revelación e inspección de los documentos - rule 31.17 (5) (b) CPR-.

\footnotetext{
${ }^{61}$ Adicionalmente, y pese a que la rule no lo contempla expresamente, la doctrina científica ha añadido un tercer requisito consistente en la exigencia de que los documentos requeridos realmente existan. Véase por todos, DI MAMBRO (2019), p. 918.

62 En este sentido se pronuncian los casos Three Rivers District Council vs. Her Majesty's Treasury [2002] EWCA Civ 1182 y Black vs. Sumitomo Corporation [2001] EWCA Civ 1819, [2002]. Al respecto véase, asimismo, HOLLANDER (2015), p. 41; ILLER (2006), p. 334; y O'HARE y BROWNE (2013), p. 520.

63 O'HARE Y BROWNE (2013), p. 521.

${ }^{64}$ GREENE (1999), pp. 249-250. En esta línea véase también DI MAMBRO (2019), p. 885.

${ }^{65}$ Véase ZUCKERMAN (2013), p. 768.

${ }^{66}$ Este contenido coincide con el de la orden judicial por la que se aprueba una pre-action disclosure prevista en los apartados 4 o y 5o de la rule 31.16 CPR, que es esencialmente aquella que se adopta en un momento previo al inicio del proceso judicial para exigir a las futuras posibles partes que revelen todos los documentos a su alcance que les sean requeridos.
} 


\subsubsection{Specify the documents or the classes of documents, which the respondent must} disclose

En primer lugar, la orden especificará los documentos o clases de documentos que el tercero (respondent) debe revelar. Esta es la cuestión más relevante sobre la que ineludiblemente deberá pronunciarse el órgano judicial por la propia finalidad de la disclosure. Ahora bien, en este punto cabe plantearse a qué documentos en concreto se refiere la norma para que efectivamente acaben siendo objeto de revelación por parte de un tercero. Esta cuestión carece de regulación expresa en esta rule, a diferencia de lo previsto para la pre-action disclosure de la rule 31.16 (3) (c) CPR - que explícitamente se remite a los documentos de la standard disclosure - lo que nos puede llevar inicialmente a considerar, y haciendo una aplicación analógica de la genérica regulación de la disclosure, que solo podrán someterse a una non-party disclosure los documentos propios de la standard disclosure, esto es, los previstos en la rule 31.6 CPR, y que se corresponden con: los documentos sobre los que pueda sostenerse la pretensión; aquellos otros que pudieran perjudicar a la propia parte que los aportase o bien a la contraparte; $y$, por último, los documentos que pudieran beneficiar a la otra parte.

Sin embargo, la doctrina judicial ha realizado una interpretación más restrictiva de los documentos cuya revelación pueden exigirse a un tercero, matizando la necesidad de que tales documentos sean relevantes, lo que no se advierte expresamente para la standard disclosure ${ }^{67}$. Para determinar dicha relevancia, el tribunal adoptará diferentes cautelas ${ }^{68}$ : en primer lugar, tendrá que llevar a cabo un análisis detallado del documento en relación con el resto de las pruebas existentes al tiempo de la presentación de la solicitud ${ }^{69}$; en segundo lugar, evitará entrar en cuestiones de fondo para determinar la relevancia de revelar el documento ${ }^{70} ; \mathrm{y}$, en tercer lugar, deberá también motivar su decisión ${ }^{71}$.

\subsubsection{Documents which are no longer in respondent's control; or in respect of which he claims a right or duty to withhold inspection}

Asimismo, en la orden judicial que acabe acordándose deberá indicarse al tercero (respondent) que especifique, en el momento de la revelación, cuando proceda, qué documentos de los requeridos ya no están bajo su control, en cuyo caso será ineludible para el tercero explicar qué les pasó ${ }^{72}$; o bien, los que aún estarlo, no deben ser inspeccionados porqué el respondent considera que tiene un derecho o un deber de no hacerlo por ser el contenido del documento privado o confidencial, pudiéndose en este caso vulnerar el derecho al respeto a la vida privada y familiar del art. 8 del Convenio Europeo de Derechos Humanos ${ }^{73}$. A pesar de ello, el tercero deberá poner tal circunstancia en conocimiento del tribunal, por escrito, para que sea el órgano judicial quien decida si finalmente los documentos tienen que ser inspeccionados ${ }^{74}$.

\footnotetext{
${ }^{67}$ Esta cuestión ha sido objeto de consideración en el caso Amercian Home Products Corporation vs. Novartis Pharmaceutical UK Ltd [2001] LTL.

${ }^{68}$ En este sentido también lo remarca SMITH (2015), p. 918.

${ }^{69}$ Véase Flood vs. Times Newspappers Ltd [2009] EWHC 411 (QB), [2009] EMLR 18; y Three Rivers District Council vs. Her Majesty's Treasury [2002] EWCA Civ 1182, [2003] 1 WLR 210.

${ }^{70}$ Véase Clark vs. Ardington Electrical Services [2001] EWCA Civ 585, LTL

${ }^{71}$ Véase Re Skyward Builders plc [2002] EWH 1788 (Ch), [2002] 2 BCLC 750.

72 Así lo ha advertido ZUCKERMAN (2013), p. 768.

${ }^{73}$ Convenio para la Protección de los Derechos Humanos y de las Libertades Fundamentales, hecho en Roma el 4 de noviembre de 1950 (https://www.echr.coe.int/Documents/Convention_SPA.pdf).

${ }^{74}$ La limitación del contenido del derecho del art. 8 del Convenio Europeo de Derecho Humanos a favor de los intereses de la justicia es objeto de análisis en el caso MS vs. Sweden [1997] 28 EHRR 313 y R vs, Local Authority in the Midlands, ex p LM [2000] 1 FCR 736. Un ejemplo de ello serían los documentos médicos que, por su carácter confidencial, se encuentran protegidos por referido art. 8 , pero que pueden acabar siendo inspeccionados si el tribunal lo acuerda al amparo del apartado segundo del mismo precepto (confróntese SMITH (2015), p. 919). En este sentido igualmente véase Andrew vs. Newsgroup Newspapers Ltd [2011] EWHC 734; y Ixis Corporate \& Investment Bank vs. Westlb AG [2007] EWHC 1852.
} 


\subsubsection{Costes de su aplicación}

La específica regulación relativa a la orden de disclosure contra tercero del artículo 31.17 CPR nada dice sobre quién debe afrontar los costes de su aplicación. En esta ocasión, el legislador inglés ha optado por regular esta cuestión en la genérica rule 46 intitulada "Costsspecial cases" y, en particular, en su apartado primero. Esta rule establece como norma que los costes de la solicitud y su cumplimiento serán a cargo de la persona contra quien se dirige la orden de disclosure, esto es, el tercero (non-party) ${ }^{75}$. Sin embargo, la discrecionalidad que tiene atribuida en este ámbito el tribunal justifica que, en virtud de la rule 46.1 (3) CPR, puedan imponerse los gastos al solicitante (applicant), teniendo en cuenta todas las circunstancias concernientes al concreto caso.

\section{Conclusiones}

Una vez examinados los elementos esenciales de la exhibición documental de terceros de la LEC y de la non-party disclosure de las CPR, llegamos a la conclusión que son más las distancias habidas entre ambas instituciones procesales que los puntos que las unen. Pasamos a continuación a destacar los de mayor relevancia.

A) Puntos de encuentro:

Primero.- Las dos instituciones procesales se desarrollan estrictamente en sede de prueba documental.

Segundo.- En ambos casos el tercero tiene la posibilidad de justificar su oposición a la exhibición del documento. De un lado, las CPR dan la oportunidad al tercero de oponerse a la exhibición del documento para su posterior inspección a través de la resolución judicial que acuerda el deber de disclosure, pues se le concede la opción de que indique los documentos que ya no están bajo su control $-\mathrm{y}$ por qué- y los que, aun estarlo, son confidenciales. De otro lado, aunque la LEC no haya previsto expresamente un trámite de oposición a tal efecto, entendemos que esta posibilidad puede tener lugar en el acto de la comparecencia prevista para que el órgano judicial pueda oír al tercero, lo que sin duda podrá influir en la decisión sobre la improcedencia de la exhibición.

Tercero.- Tanto en el sistema español como en el inglés, la parte deberá justificar la trascendencia del documento objeto de exhibición por parte del tercero. Así, mientras que esta trascendencia se desprende del tenor literal de la LEC como uno de los requisitos propios de la exhibición documental de terceros, la norma procesal inglesa no la contempla; sin embargo, esta carencia la suple la doctrina judicial al exigir la necesidad de que los documentos requeridos a terceros sean relevantes.

Cuarto.- En última instancia es el órgano judicial, en virtud de la discrecionalidad que tiene conferida en ambos ordenamientos jurídicos, a quien corresponde decidir sobre la exhibición de documentos en poder de terceros.

B) Puntos de desencuentro

Primero.- El legislador español solo prevé la obligatoriedad de la exhibición documental de terceros para las entidades públicas. En cambio, el hecho de que legislador británico haya extendido el deber de disclosure a los terceros justifica que, en este caso, se trate también de una obligación para el tercero.

Segundo.- La regulación que las CPR disponen para la disclosure against non-parties es más completa que la prevista en la LEC para la exhibición de documentos por parte de terceros.

Tercero.- Ambos ordenamientos jurídicos coinciden al establecer unos requisitos mínimos para acordar la exhibición documental por un tercero. Sin embargo, observamos que la disclosure against non-parties va más allá de la simple aportación de los documentos que

${ }^{75}$ SMITH (2015), p. 919, destacan que en el caso de la pre-action disclosure los costes también son asumidos por la persona contra quien se dirige la orden (respondent). 
favorezcan al propio solicitante al incluir, también, aquellos otros que afecten negativamente a cualquiera de las partes con el objetivo último de alcanzar un resultado justo. De ello se desprende que el sistema anglosajón es más garantista en este extremo por cuanto apuesta por la incorporación al proceso de todos los elementos probatorios en aras a alcanzar, como se desprende de los overriding principles (rule 1.1 CPR) una resolución justa, eficiente, rápida y económica del conflicto ${ }^{76}$.

Cuarto.- Del deber de disclosure de terceros se excluyen aquellos documentos que sean privados o confidenciales. No obstante, el legislador español tan solo ha previsto esta exclusión para las entidades oficiales, dejándola de regular para el resto de los terceros.

Quinto.- El último punto disonante hace referencia a la asunción de los costes derivados de la exhibición documental. En la disclosure generalmente asume los gastos el tercero. En cambio, en este caso es más adecuada la respuesta de la LEC pues, pese a no contemplar específicamente quien asume los gastos en los preceptos relativos a la exhibición documental de terceros entendemos que, en la medida que es un gasto del proceso, son de aplicación los arts. 241 y 394 LEC, de cuya interpretación conjunta resulta que, por regla general, será la parte que haya visto rechazadas todas sus pretensiones $-\mathrm{y}$ no el tercero- quien deba asumir este coste, en virtud del principio de vencimiento.

\section{BIBLIOGRAFÍA CITADA}

ABel LlUCH, XAVIER (2010): La prueba documental (Barcelona, Editorial J.M. Bosch).

Ashfield, Evan; Browne, Julie; Jolly, Peter.; Keane, Adrian; laurenti, Lisa; lidbetter, Andrew; OWens, Alan; Petroldi, Anna; Rose, William.; Scott, Charles; Weiniger, Matthew (2015): Blackstone's Civil Practice. The commentary 2015, 4a edición (Oxford, Editorial Oxford University Press).

BLACKIE, KIM Y BATES, JOHN (2014): Civil Litigation in Practice (Newcastle, Editorial Northumbria Law Press).

Browne, KeVIN y CATLow, Margaret (2014): Civil Litigation (Guildford, Editorial College of Law Publishing).

CASANOVA MARTí, Roser (2019): “De los documentos privados”, en: Izquierdo, Pablo; Picó, Joan y Adán, Federico (Dirs.), Todas las preguntas y respuestas sobre de Ley de Enjuiciamiento Civil (Madrid, Editorial Wolters Kluwer - Bosch), pp. 760-777.

CUNNINGHAM-HILL, SUSAN Y ELDER, KAREN (2013): Civil Litigation Handbook (Oxford, Editorial Oxford University Press).

De Prada Rodríguez, Mercedes y Muñoz Rojo, Roberto (2014): El proceso civil inglés (Granada, Editorial Comares).

DI MAMBRO, DAVID (2019): The Civil Court Practice 2019 (London, Editorial LexisNexis), volumen 1.

Greene, DAVID (1999): The New Civil Procedure Rules (London, Editorial Butterworths).

Hill, Robert; Wood, Helen y Fine, SUzAnne (2005): A Practical Guide to Civil Litigation (Bristol, Editorial Jordans).

Hollander, Charles (2015): Documentary Evidence, 12a edición (London, Sweet \& Maxwell).

ILLER, MARTIN (2006): Civil Evidence: The Essential Guide (London, Editorial Thomson Sweet \& Maxwell).

\footnotetext{
${ }^{76}$ Según esta norma, las CPR son un nuevo código procesal cuyo objetivo primordial es permitir al tribunal resolver los casos de forma justa y por un coste proporcionado.
} 
Matthews, Paul y Malek, Hodge M. (2017): Disclosure, 5a edición (London, Editorial Sweet\&Maxwell - Thomson Routers).

O'HARE, JOHN Yy Browne, KeVIN (2013): Civil Litigation (London, Editorial Sweet and Maxwell Thomson Reuters).

PeÑA, CRISTÓBAL (2017): "Traduciendo el discovery al civil law chileno: Su aporte a los procesos de reforma procesal civil”, en: lus et Praxis (Vol. 23, № 2), pp. 79-120.

PICÓ I JUNOY, JOAN (2012): El principio de la buena fe procesal, 2ª edición (Barcelona, Editorial J.M. Bosch).

(2014): “Los procesos declarativos: Disposiciones comunes (arts. 248 a 334)", en: Corbal, Jesús; Izquierdo, Pablo y Picó, Joan (Dirs.), Práctica procesal civil, Brocá-Majada-Corbal, 23a edición (Barcelona, Editorial Bosch), tomo V, pp. 3948-3952.

SMITH, Graeme (2015): Civil Court Service (Bristol, Editorial Jordan Publishing).

ZUCKERMAN, AdRIAN (2013): Zuckerman on Civil Procedure. Principles of Practice (London, Sweet \& Maxwell - Thomson Reuters).

\section{JURISPRUDENCIA CITADA}

MS VS. SWEDEN [1997] 28 EHRR 313 Y R VS, LOCAL AUTHORITY IN THE MIDLANDS, EX P LM [2000] 1 FCR 736 (United Kingdom).

CLARK VS. ARdington EleCtrical SeRVICES [2001] EWCA CIV 585, LTL (United Kingdom).

amercian home Products Corporation vs. Novartis Pharmaceutical UK Ltd [2001] LTL ((United Kingdom).

BLACK VS. SUMITOMO CORPORATION [2001] EWCA CIV 1819, [2002] (United Kingdom).

RE SKYWARD BUILDERS PLC [2002] EWH 1788 (CH), [2002] 2 BCLC 750 (United Kingdom).

THREe RIVERS DISTRICT CounCIL VS. HeR MAJeStY's TREASURY [2002] EWCA CIV 1182 (United Kingdom).

SAP DE BADAJOZ (SECCIÓN 3a), 88/2003, DE 18 DE FEBRERO [JUR 2003\162412] (España).

SAP DE A CORUÑa (SECCIÓN 6a), 157/2005, DE 22 dE MARZO [JUR 2006/93886] (España).

TAJIK ALUMINIUM PLANT VS HYDRO ALUMINIUM AS [2005] EWCA CIV 1218 (United Kingdom).

IXIS CORPORATE \& INVESTMENT BANK VS. WESTLB AG [2007] EWHC 1852 (United Kingdom).

STS 479/2008, SALA DE LO CIVIL, DE 3 DE JUNIO [ECLI: ES:TS:2008:4309] (España).

FLOOD VS. TIMES NEWSPAPPERS LTD [2009] EWHC 411 (QB), [2009] EMLR 18 ((United Kingdom).

SAP DE PALENCIA (SECCIÓN 1a), 247/2010, DE 20 DE SEPTIEMBRE [ECLI:ES:APP:2010:450] (España).

SAP DE HuelVa (SECCIÓN 1a)), 194/2010, de 26 DE OCTUBRe [ECLI:ES:APH:2010:682] (España).

ANDREW VS. NEWSGROUP NEWSPAPERS LTD [2011] EWHC 734 (United Kingdom).

SJMERCANTIL NÚM. 7 DE BARCELONA, 357/2011, DE 16 DE DICIEMBRE [ECLI:ES:JMB:2011:205] (España).

SAP DE SALAMANCA (SECCIÓN 1ą), 36/2012, DE 30 DE ENERo [ECLI: ECLI:ES:APSA:2012:37] (España).

SAP DE GRANADA (SECCIÓN 5a)), 412/2012, DE 26 DE OCTUBRE [ECLI:ES:APGR:2012:2300] (España).

SAP DE OVIEDO (SECCIÓN 6a)), 126/2014, DE 2 DE JUNIO [ECLI:ES:APO:2014:1419] (España). 
SAP DE BARCELONA (SECCIÓN 11a), 211/2015, DE 23 DE JULIO [ECLI:ES:APB:2015:8022] (España).

SAP DE BILBAO (SECCIÓN 4a), 366/2017, DE 17 DE MAYO [ECLI: ES:APBI:2017:997] (España).

STS 644/2017, SALA DE LO CIVIL, DE 24 DE NOVIEMBRE [ECLI: ES:TS:2017:4119] (España).

SAP BARCELONA, 848/2017, DE 18 DE DICIEMBRE [ECLI:ES:APB:2017:11973] (España).

SAP dE BARCELONA (SECCIÓN 15a), 423/2018, DE 25 DE JUNIO [ECLI:ES:APB:2018:6540] (España).

\section{NORMAS JURÍDICAS CITADAS}

CONSTITUCIÓn ESPAÑOLA. Boletín Oficial del Estado, 29 de diciembre de 1978, núm. 311.

Rule № 3132, The Civil Procedure Rules. UK Statutory Instruments, 10 de diciembre de 1998.

ESPAÑA. LeY №1/2000 de ENJUICIAMIENTO CiVIL. Boletín Oficial del Estado, 8 de enero de 2000, núm. 7. 